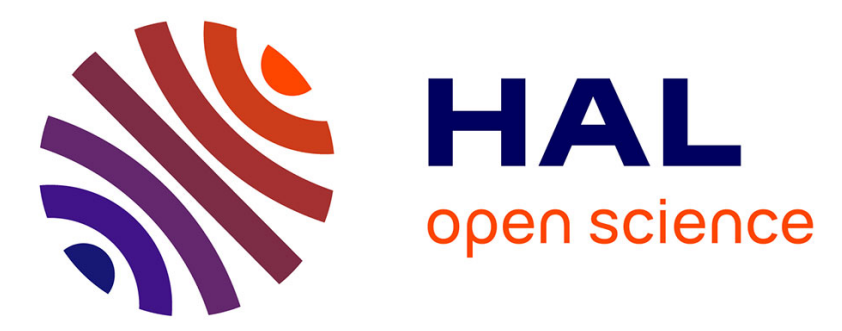

\title{
Well-posedness and asymptotic stability results for a viscoelastic plate equation with a logarithmic nonlinearity
}

\author{
Mohammad M Al-Gharabli, Aissa Guesmia, Salim Messaoudi
}

\section{To cite this version:}

Mohammad M Al-Gharabli, Aissa Guesmia, Salim Messaoudi. Well-posedness and asymptotic stability results for a viscoelastic plate equation with a logarithmic nonlinearity. Applicable Analysis, 2020, 99, pp.50 - 74. 10.1080/00036811.2018.1484910 . hal-02891552

\section{HAL Id: hal-02891552 \\ https://hal.science/hal-02891552}

Submitted on 15 Aug 2020

HAL is a multi-disciplinary open access archive for the deposit and dissemination of scientific research documents, whether they are published or not. The documents may come from teaching and research institutions in France or abroad, or from public or private research centers.
L'archive ouverte pluridisciplinaire HAL, est destinée au dépôt et à la diffusion de documents scientifiques de niveau recherche, publiés ou non, émanant des établissements d'enseignement et de recherche français ou étrangers, des laboratoires publics ou privés. 


\title{
WELL-POSEDNESS AND ASYMPTOTIC STABILITY RESULTS FOR A VISCOELASTIC PLATE EQUATION WITH A LOGARITHMIC NONLINEARITY
}

\author{
Mohammad M. Al-Gharabli(1), Aissa Guesmia ${ }^{(2)}$ and Salim A. Messaoudi ${ }^{(3)}$ \\ (1) King Fahd University of Petroleum and Minerals \\ The Preparatory Year Program, Department of Mathematics \\ Dhahran 31261, Saudi Arabia. \\ E-mail: mahfouz@kfupm.edu.sa \\ (2) Institut Elie Cartan de Lorraine, UMR 7502 \\ Université de Lorraine, 3 Rue Augustin Fresnel \\ BP 45112, 57073 Metz Cedex 03, France. \\ E-mail: aissa.guesmia@univ-lorraine.fr \\ (3) King Fahd University of Petroleum and Minerals \\ Department of Mathematics and Statistics \\ Dhahran 31261, Saudi Arabia. \\ E-mail: messaoud@kfupm.edu.sa
}

\begin{abstract}
In this paper, we consider a viscoelastic plate equation with a velocity-dependent material density and a logarithmic nonlinearity. Using the Faedo-Galaerkin approximations and the multiplier method, we establish the existence of the solutions of the problem and we prove an explicit and general decay rate result. These results extend and improve many results in the literature.
\end{abstract}

\section{INTRODUCTION}

In this paper, we deal with the existence and decay of solutions of the following plate problem:

$$
\left\{\begin{array}{lc}
\left|u_{t}\right|^{\rho} u_{t t}+\Delta^{2} u+\Delta^{2} u_{t t}-\int_{0}^{t} g(t-s) \Delta^{2} u(s) d s=k u \ln |u|, & \text { in } \Omega \times(0, \infty), \\
u(x, t)=\frac{\partial u}{\partial \nu}(x, t)=0, & \text { in } \partial \Omega \times(0, \infty), \\
u(x, 0)=u_{0}(x), \quad u_{t}(x, 0)=u_{1}(x), & \text { in } \Omega,
\end{array}\right.
$$

where $\Omega$ is a bounded domain of $\mathbb{R}^{2}$, with a smooth boundary $\partial \Omega$, $\nu$ is the unit outer normal to $\partial \Omega$ and $\rho$ and $k$ are positive constants. The kernel $g$ is satisfying some conditions to be specified later.

1.1. Problems with a velocity-dependent material density. Cavalcanti et al. 6] considered

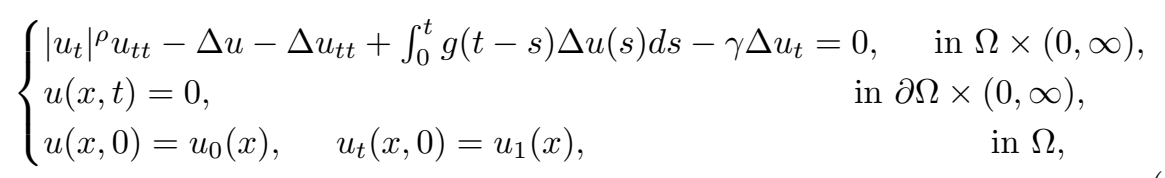


where $\Omega$ is a bounded domain in $\mathbb{R}^{n}, n \geq 1$, with a smooth boundary $\partial \Omega, \rho$ is a positive real number satisfying some conditions and $g$ is a positive exponentially decaying function. They established a global existence result when the constant $\gamma \geq 0$, and an exponential decay result for the case $\gamma>0$. Messaoudi and Tatar 33 extended this decay result to the case where a source term is competing with the viscoelastic and the strong damping. In the absence of the strong damping $(\gamma=0)$, Messaoudi and Tatar 34, 35] studied (1.2) and showed that the viscoelastic damping is strong enough to drive the system uniformly to rest. Precisely, they showed that the energy of the solution decays exponentially (resp. polynomially) if $g$ decays exponentially (resp. polynomially). Later, Han and Wang [18 considered 1.2 for $\gamma=0$ and with a relaxation function of more general decay type and established, similarly to the work of Messaoudi [30, 31, a general decay result in which the usual exponential and the polynomial decay are only special cases. Liu [27] considered 1.2 , for $\gamma=0$, and in the presence of a source term. He established a general decay result similar to the one in [18. In [28, Liu studied the problem

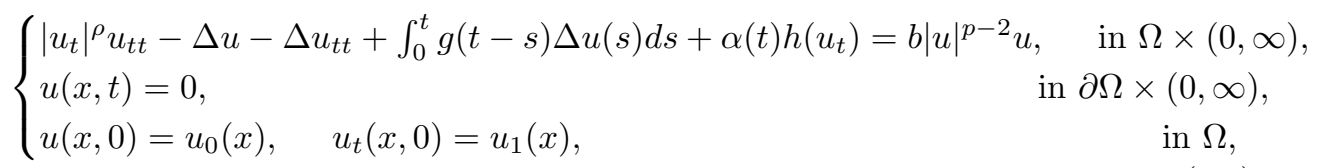

and proved, without imposing growth conditions on $h$, a general decay result which depends on the behavior of $g, \alpha$ and $h$. Messaoudi and Mustafa [36] studied 1.2 for relaxation functions satisfying

$$
g^{\prime}(t) \leq-H(g(t))
$$

where $H \in \mathbb{C}^{1}\left(\mathbb{R}^{+}\right)$, with $H(0)=0$ and $H$ is linear or strictly increasing and strictly convex function $\mathbb{C}^{2}$ near the origin. They obtained an explicit and general relation between the decay rate for the energy and that of the relaxation function $g$ without imposing restrictive assumptions on the behavior of $g$ at infinity. Recently, Cavalcanti et al. 8] considered (1.2), with $\gamma=0$, and a relaxation function satisfying (1.4). In addition, they required

$$
\liminf _{x \rightarrow 0^{+}}\left\{x^{2} H^{\prime \prime}(x)-x H^{\prime}(x)+H(x)\right\} \geq 0
$$

and some other condition and proved that the energy uniformly decays to zero with the rate that is determined from the solutions of the ODE quantifying the behavior of $g(t)$.

Very recently, Messaoudi and Al-Khulaifi 32 considered (1.2), with $\gamma=0$, where the relaxation function satisfies $(2.2)$ below and established an optimal and general decay result.

1.2. Plate Problems. Concerning the study of plates, Lagnese 23] studied a viscoelastic plate equation and showed that the energy decays to zero as time goes to infinity by intorducing a dissipative mechanism on the boundary of the system. Rivera et al. 37. proved that the first and second order energy, associated with the solutions of the viscoelastic plate equation, decay exponentially provided that the kernel of the memory also decays exponentially. Komornik [21] investigated the energy decay of a plate model under weak growth assumptions on the feedback function. Messaoudi [29] studied the following problem: 


$$
\begin{cases}u_{t t}+\Delta^{2} u+\left|u_{t}\right|^{m-2} u_{t}=|u|^{p-2} u, & \text { in } Q_{T}=\Omega \times(0, T), \\ u=\frac{\partial u}{\partial \nu}=0, & \text { on } \Gamma_{T}=\partial \Omega \times(0, T), \\ u(x, 0)=u_{0}(x), u_{t}(x, 0)=u_{1}(x), & \text { in } \Omega,\end{cases}
$$

and established an existence result and showed that the solution continues to exist globally if $m \geq p$, and blows up in finite time if $m<p$ and the initial energy is negative. This result was later improved by Chen and Zhou [11.

For boundary damping, Santos and Junior [38] studied the stability of the following problem:

$$
\begin{cases}u_{t t}+\Delta^{2} u=0, & \text { in } \Omega \times(0, \infty), \\ u=\frac{\partial u}{\partial \nu}=0, & \text { on } \Gamma_{0} \times(0, \infty), \\ -u+\int_{0}^{t} g_{1}(t-s) \beta_{1} u(s) d s=0, & \text { on } \Gamma_{1} \times(0, \infty), \\ \frac{\partial u}{\partial \nu}+\int_{0}^{t} g_{2}(t-s) \beta_{2} u(s) d s=0, & \text { on } \Gamma_{2} \times(0, \infty), \\ u(0, x)=u_{0}(x), \quad u_{t}(0, x)=u_{1}(x), & \text { in } \Omega,\end{cases}
$$

where

$$
\beta_{1} u=\Delta u+(1-\mu) B_{1} u \quad \text { and } \quad \beta_{2} u=\frac{\partial \Delta u}{\partial \mu}+(1-\mu) \frac{\partial B_{2} u}{\partial \eta}
$$

with

$B_{1} u=2 \nu_{1} \nu_{2} u_{x y}-\nu_{1}^{2} u_{y y}-\nu_{2}^{2} u_{x x} \quad$ and $\quad B_{2} u=\left(\nu_{1}-\nu_{2}\right) u_{x y}+\nu_{1} \nu_{2}\left(u_{y y}-u_{x x}\right)$.

For more results in this direction, see [3, 16, 19, 22, 25].

1.3. Problems with Logarithmic Nonlinearity. The logarithmic nonlinearity is of much interest in physics, since it appears naturally in inflation cosmology and supersymmetric filed theories, quantum mechanics and nuclear physics [1, 12. This type of problems has applications in many branches of physics such as nuclear physics, optics and geophysics [2, 4, 13. Birula and Mycielski [4, 5] studied the following problem:

$$
\begin{cases}u_{t t}-u_{x x}+u-\varepsilon u \ln |u|^{2}=0, & \text { in }[a, b] \times(0, T), \\ u(a, t)=u(b, t)=0, & \text { in }(0, T), \\ u(x, 0)=u_{0}(x), \quad u_{t}(x, 0)=u_{1}(x), & \text { in }[a, b],\end{cases}
$$

which is a relativistic version of logarithmic quantum mechanics and can also be obtained by taking the limit $p \rightarrow 1$ for the $p$-adic string equation [14, 39. In 9, Cazenave and Haraux considered

$$
u_{t t}-\Delta u=u \ln |u|^{k}, \quad \text { in } \mathbb{R}^{3}
$$

and established the existence and uniqueness of the solution for the Cauchy problem. Gorka [13] used some compactness arguments and obtained the global existence of weak solutions, for all

$$
\left(u_{0}, u_{1}\right) \in H_{0}^{1}([a, b]) \times L^{2}([a, b]),
$$

to the initial-boundary value problem (1.8) in the one-dimensional case. Bartkowski and Gorka [2] proved the existence of classical solutions and investigated the weak 
solutions for the corresponding one-dimensional Cauchy problem for equation (1.8). Hiramatsu et al. 20] introduced the following equation:

$$
u_{t t}-\Delta u+u+u_{t}+|u|^{2} u=u \ln |u|
$$

to study the dynamics of Q-ball in theoretical physics and presented a numerical study. However, there was no theoretical analysis for the problem. In [17, Han proved the global existence of weak solutions, for all

$$
\left(u_{0}, u_{1}\right) \in H_{0}^{1}(\Omega) \times L^{2}(\Omega),
$$

to the initial boundary value problem 1.9 in $\mathbb{R}^{3}$.

In this paper, we are concerned with the well-posedness and stability of the plate problem (1.1) with kernels $g$ having an arbitrary growth at infinity (condition 2.2) below). The obtained stability results improve and generalize many results in the literature.

This paper is organized as follows. In section 2, we present some notations and material needed for our work. In section 3, we establish the local existence of the solutions of the problem. The global existence and the decay results are presented in section 4 and section 5 , respectively.

\section{Preliminaries}

In this section, we present some material needed for the proof of our results. We use the standard Lebesgue space $L^{2}(\Omega)$ and Sobolev space $H_{0}^{2}(\Omega)$ with their usual scalar products and norms. Throughout this paper, c is used to denote a generic positive constant.

We consider the following hypotheses:

(A1) $g: \mathbb{R}^{+} \rightarrow \mathbb{R}^{+}$is a $C^{1}$ - nonincreasing function satisfying

$$
g(0)>0, \quad 1-\int_{0}^{\infty} g(s) d s=\ell>0 .
$$

(A2) There exist a nonincreasing differentiable function $\xi: \mathbb{R}^{+} \rightarrow \mathbb{R}^{+}$, with $\xi(0)>0$, and a constant $1 \leq p<\frac{3}{2}$ such that

$$
g^{\prime}(t) \leq-\xi(t) g^{p}(t), \forall t \in \mathbb{R}^{+} .
$$

(A3) The constant $k$ in 1.1 is such that

$$
0<k<k_{0}=\frac{2 \pi \ell e^{3}}{c_{p}},
$$

where $c_{p}$ is the smallest positive number satisfying

$$
\|\nabla u\|_{2}^{2} \leq c_{p}\|\Delta u\|_{2}^{2}, \quad \forall u \in H_{0}^{2}(\Omega),
$$

where $\|\cdot\|_{2}=\|\cdot\|_{L^{2}(\Omega)}$.

The energy functional associated with problem (1.1) is

$$
\begin{aligned}
E(t)=\frac{1}{\rho+2}\left\|u_{t}\right\|_{\rho+2}^{\rho+2} & +\frac{1}{2}\left(\left(1-\int_{0}^{t} g(s) d s\right)\|\Delta u\|_{2}^{2}+\left\|\Delta u_{t}\right\|_{2}^{2}-k \int_{\Omega} u^{2} \ln |u| d x\right) \\
& +\frac{k}{4}\|u\|_{2}^{2}+\frac{1}{2}(g o \Delta u),
\end{aligned}
$$


where

$$
(g \circ \Delta u)(t)=\int_{0}^{t} g(t-s)\|\Delta u(s)-\Delta u(t)\|_{2}^{2} d s .
$$

Direct differentiation of 2.4, using 1.1), leads to

$$
E^{\prime}(t)=\frac{1}{2}\left(g^{\prime} o \Delta u\right)(t)-\frac{1}{2} g(t)\|\Delta u\|_{2}^{2} \leq \frac{1}{2}\left(g^{\prime} o \Delta u\right)(t) \leq 0 .
$$

Lemma 2.1. [10, 15] (Logarithmic Sobolev inequality) Let $u$ be any function in $H_{0}^{1}(\Omega)$ and $a>0$ be any number. Then

$$
\int_{\Omega} u^{2} \ln |u| d x \leq \frac{1}{2}\|u\|_{2}^{2} \ln \|u\|_{2}^{2}+\frac{a^{2}}{2 \pi}\|\nabla u\|_{2}^{2}-(1+\ln a)\|u\|_{2}^{2} .
$$

Corollary 2.2. Let $u$ be any function in $H_{0}^{2}(\Omega)$ and $a>0$ be any number. Then

$$
\int_{\Omega} u^{2} \ln |u| d x \leq \frac{1}{2}\|u\|_{2}^{2} \ln \|u\|_{2}^{2}+\frac{c_{p} a^{2}}{2 \pi}\|\Delta u\|_{2}^{2}-(1+\ln a)\|u\|_{2}^{2} .
$$

Lemma 2.3. 9] (Logarithmic Gronwall inequality) Let $C>0, \gamma \in L^{1}\left(0, T ; \mathbb{R}^{+}\right.$) and assume that the function $w:[0, T] \rightarrow[1, \infty)$ satisfies

$$
w(t) \leq C\left(1+\int_{0}^{t} \gamma(s) w(s) \ln (w(s)) d s\right), \quad \forall t \in[0, T] .
$$

Then

$$
w(t) \leq C \exp \left(C \int_{0}^{t} \gamma(s) d s\right), \quad \forall t \in[0, T] .
$$

Lemma 2.4. Let $\epsilon_{0} \in(0,1)$. Then there exists $d_{\varepsilon_{0}}>0$ such that

$$
s|\ln s| \leq s^{2}+d_{\epsilon_{0}} s^{1-\epsilon_{0}}, \forall s>0 .
$$

Proof. Let $r(s)=s^{\epsilon_{0}}(|\ln s|-s)$. Notice that $r$ is continuous on $(0, \infty)$ and its limit at $0^{+}$is $0^{+}$, and its limit at $\infty$ is $-\infty$. Then $r$ has a maximum $d_{\epsilon_{0}}$ on $(0, \infty)$, so 2.10 holds.

\section{LOCAL EXISTENCE}

In this section, we state and prove the local existence result for problem 1.1.

Definition 3.1. Let $T>0$. A function

$$
u \in C^{1}\left([0, T], H_{0}^{2}(\Omega)\right)
$$

is called a weak solution of 1.1 on $[0, T]$ if

$$
\left\{\begin{array}{c}
\int_{\Omega}\left|u_{t}\right|^{\rho} u_{t t}(x, t) w(x) d x+\int_{\Omega} \Delta u(x, t) \Delta w(x) d x+\int_{\Omega} \Delta u_{t t} \Delta w d x \\
-\int_{\Omega} \Delta w(x) \int_{0}^{t} g(t-s) \Delta u(s) d s d x=k \int_{\Omega} w(x) u(x, t) \ln |u(x, t)| d x, \forall w \in H_{0}^{2}(\Omega), \\
u(x, 0)=u_{0}(x), \quad u_{t}(x, 0)=u_{1}(x) .
\end{array}\right.
$$

Theorem 3.2. Assume that $(A 1)-(A 3)$ hold and let $\left(u_{0}, u_{1}\right) \in H_{0}^{2}(\Omega) \times H_{0}^{2}(\Omega)$. Then problem 1.1) has a weak solution on $[0, T]$. 
Proof. To establish the existence of a solution to problem (1.1), we use the FaedoGalerkin approximations. Let $\left\{w_{j}\right\}_{j=1}^{\infty}$ be an orthogonal basis of the "separable" space $H_{0}^{2}(\Omega)$. Let $V_{m}=\operatorname{span}\left\{w_{1}, w_{2}, \ldots, w_{m}\right\}$ and let the projections of the initial data on the finite dimensional subspace $V_{m}$ be given by

$$
u_{0}^{m}(x)=\sum_{j=1}^{m} a_{j} w_{j}(x), \quad u_{1}^{m}(x)=\sum_{j=1}^{m} b_{j} w_{j}(x),
$$

where

$$
u_{0}^{m} \rightarrow u_{0} \text { in } H_{0}^{2}(\Omega) \text { and } \quad u_{1}^{m} \rightarrow u \text { in } H_{0}^{2}(\Omega) \text {, as } m \rightarrow \infty .
$$

We search for an approximate solution

$$
u^{m}(x, t)=\sum_{j=1}^{m} h_{j}^{m}(t) w_{j}(x)
$$

of the approximate problem in $V_{m}$

$$
\left\{\begin{array}{c}
\int_{\Omega}\left(\left|u_{t}^{m}\right|^{\rho} u_{t t}^{m} w+\Delta u^{m} \Delta w+\Delta u_{t t}^{m} \Delta w-\int_{0}^{t} g(t-s) \Delta u^{m}(s) \Delta w d s\right) d x \\
=k \int_{\Omega} w u^{m} \ln \left|u^{m}\right| d x, \forall w \in V_{m}, \\
u^{m}(0)=u_{0}^{m}=\sum_{j=1}^{m}\left(u_{0}, w_{j}\right) w_{j}, \\
u_{t}^{m}(0)=u_{1}^{m}=\sum_{j=1}^{m}\left(u_{1}, w_{j}\right) w_{j} .
\end{array}\right.
$$

This leads to a system of ODEs for unknown functions $h_{j}^{m}(t)$. Based on standard existence theory for ODE, one can obtain functions

$$
h_{j}:\left[0, t_{m}\right) \rightarrow \mathbb{R}, j=1,2, \ldots, m,
$$

which satisfy $(3.3)$ in a maximal interval $\left[0, t_{m}\right), t_{m} \in(0, T]$. Next, we show that $t_{m}=T$ and that the local solution is uniformly bounded independently of $m$ and $t$. For this purpose, let $w=u_{t}^{m}$ in (3.3) and integrate by parts to obtain

$$
\frac{d}{d t} E^{m}(t) \leq \frac{1}{2}\left(g^{\prime} o \Delta u^{m}\right) \leq 0
$$

where

$$
\begin{gathered}
E^{m}(t)=\frac{1}{\rho+2}\left\|u_{t}^{m}\right\|_{\rho+2}^{\rho+2}+\frac{1}{2}\left(\left\|\Delta u_{t}^{m}\right\|_{2}^{2}+\left(1-\int_{0}^{t} g(s) d s\right)\left\|\Delta u^{m}\right\|_{2}^{2}+\left(g o \Delta u^{m}\right)(t)\right) \\
+\frac{k}{4}\left\|u^{m}\right\|_{2}^{2}-\frac{k}{2} \int_{\Omega}\left|u^{m}\right|^{2} \ln \left|u^{m}\right| d x .
\end{gathered}
$$

From (3.4), we have

$$
E^{m}(t) \leq E^{m}(0), \quad \forall t \geq 0 .
$$

The last inequality together with the Logarithmic Sobolev inequality leads to

$$
\begin{gathered}
\left\|u_{t}^{m}\right\|_{\rho+2}^{\rho+2}+\left\|\Delta u_{t}^{m}\right\|_{2}^{2}+\left(\ell-\frac{k a^{2} c_{p}}{2 \pi}\right)\left\|\Delta u^{m}\right\|_{2}^{2}+\left[\frac{k}{2}+k(1+\ln a)\right]\left\|u^{m}\right\|_{2}^{2}+g o \Delta u^{m} \\
\leq C+\left\|u^{m}\right\|_{2}^{2} \ln \left\|u^{m}\right\|_{2}^{2},
\end{gathered}
$$

where $C=2 E^{m}(0)$. Choosing

$$
e^{-\frac{3}{2}}<a<\sqrt{\frac{2 \pi \ell}{k c_{p}}}
$$


will make

$$
\ell-\frac{k a^{2} c_{p}}{2 \pi}>0
$$

and

$$
\frac{k}{2}+k(1+\ln a)>0 .
$$

This selection is possible thanks to $(A 3)$. So, we get

$$
\left\|u_{t}^{m}\right\|_{\rho+2}^{\rho+2}+\left\|\Delta u_{t}^{m}\right\|_{2}^{2}+\left\|\Delta u^{m}\right\|_{2}^{2}+\left\|u^{m}\right\|_{2}^{2}+g_{o} \Delta u^{m} \leq c\left(1+\left\|u^{m}\right\|_{2}^{2} \ln \left\|u^{m}\right\|_{2}^{2}\right) .
$$

Let us note that

$$
u^{m}(., t)=u^{m}(., 0)+\int_{0}^{t} \frac{\partial u^{m}}{\partial s}(., s) d s .
$$

Then, using Cauchy-Schwarz' inequality, we get

$$
\begin{aligned}
\left\|u^{m}(t)\right\|_{2}^{2} & \leq 2\left\|u^{m}(0)\right\|_{2}^{2}+2\left\|\int_{0}^{t} \frac{\partial u^{m}}{\partial s}(s) d s\right\|_{2}^{2} \\
& \leq 2\left\|u^{m}(0)\right\|_{2}^{2}+2 T \int_{0}^{t}\left\|u_{t}^{m}(s)\right\|_{2}^{2} d s,
\end{aligned}
$$

hence, inequality (3.8) gives

$$
\left\|u^{m}\right\|_{2}^{2} \leq 2\left\|u^{m}(0)\right\|_{2}^{2}+2 T c\left(1+\int_{0}^{t}\left\|u^{m}\right\|_{2}^{2} \ln \left\|u^{m}\right\|_{2}^{2} d s\right) .
$$

If we put $C_{1}=\max \left\{2 T c, 2\|u(0)\|_{2}^{2}\right\}, 3.10$ leads to

$$
\left\|u^{m}\right\|_{2}^{2} \leq 2 C_{1}\left(1+\int_{0}^{t}\left\|u^{m}\right\|_{2}^{2} \ln \left(\left\|u^{m}\right\|_{2}^{2}\right) d s\right) .
$$

Because $C_{1} \geq 0$, we get

$$
\left\|u^{m}\right\|_{2}^{2} \leq 2 C_{1}\left(1+\int_{0}^{t}\left(C_{1}+\left\|u^{m}\right\|_{2}^{2}\right) \ln \left(C_{1}+\left\|u^{m}\right\|_{2}^{2}\right) d s\right) .
$$

Applying the Logarithmic Gronwall inequality to the last inequality, we obtain the following estimate:

$$
\left\|u^{m}\right\|_{2}^{2} \leq 2 C_{1} e^{2 C_{1} T}=C_{2} .
$$

Hence, from inequality 3.8 it follows that

$$
\left(g \circ \Delta u^{m}\right)(t)+\left\|u_{t}^{m}\right\|_{\rho+2}^{\rho+2}+\left\|\Delta u_{t}^{m}\right\|_{2}^{2}+\left\|\Delta u^{m}\right\|_{2}^{2}+\left\|u^{m}\right\|_{2}^{2} \leq c\left(1+C_{2} \ln C_{2}\right)=C_{3} .
$$

This implies

$$
\sup _{t \in\left(0, t_{m}\right)}\left[\left(g \circ \Delta u^{m}\right)(t)+\left\|u_{t}^{m}\right\|_{\rho+2}^{\rho+2}+\left\|\Delta u_{t}^{m}\right\|_{2}^{2}+\left\|\Delta u^{m}\right\|_{2}^{2}+\left\|u^{m}\right\|_{2}^{2}\right] \leq C_{3} .
$$

So, the approximate solution is uniformly bounded independent of $m$ and $t$. Therefore, we can extend $t_{m}$ to $T$. 
Substituting $w=u_{t t}^{m}$ in 3.3 and using Young's and Cauchy-Schwarz' inequalities, we obtain

$$
\begin{aligned}
\int_{\Omega}\left|u_{t}^{m}\right|^{\rho}\left|u_{t t}^{m}\right|^{2} d x+\left\|\Delta u_{t t}^{m}\right\|_{2}^{2}= & -\int_{\Omega} \Delta u^{m} \Delta u_{t t}^{m} d x+\int_{\Omega} \int_{0}^{t} g(t-s) \Delta u^{m}(s) \Delta u_{t t}^{m}(t) d s d x \\
& +k \int_{\Omega} u_{t t}^{m} u^{m} \ln \left|u^{m}\right| d x \\
\leq \delta\left\|\Delta u_{t t}^{m}\right\|_{2}^{2} & +\frac{1}{4 \delta}\left(\int_{0}^{t} g(t-s)\left\|\Delta u^{m}(s)\right\|_{2} d s\right)^{2}+\delta\left\|\Delta u_{t t}^{m}\right\| \\
& +\frac{1}{4 \delta}\left\|\Delta u^{m}(t)\right\|^{2}+k \int_{\Omega} u_{t t}^{m} u^{m} \ln \left|u^{m}\right| d x .
\end{aligned}
$$

To estimate the last term in the right-hand side of 3.12 , we apply 2.10 with $\varepsilon_{0}=$ $\frac{1}{2}$ and use repeatedly Young's, Cauchy-Schwarz' and the embedding inequalities as follows

$$
\begin{aligned}
k \int_{\Omega} u_{t t}^{m} u^{m} \ln \left|u^{m}\right| d x & \leq c \int_{\Omega} u_{t t}^{m}\left(\left|u^{m}\right|^{2}+d_{2} \sqrt{u^{m}}\right) d x \\
& \leq c\left(\delta \int_{\Omega}\left|u_{t t}^{m}\right|^{2} d x+\frac{1}{4 \delta} \int_{\Omega}\left(\left|u^{m}\right|^{2}+d_{2} \sqrt{u^{m}}\right)^{2} d x\right) \\
& \leq c \delta\left\|\Delta u_{t t}^{m}\right\|_{2}^{2}+\frac{c}{4 \delta}\left(\int_{\Omega}\left|u^{m}\right|^{4} d x+\int_{\Omega}\left|u^{m}\right| d x\right) \\
& \leq c \delta\left\|\Delta u_{t t}^{m}\right\|_{2}^{2}+\frac{c}{4 \delta}\left(\left\|\Delta u^{m}\right\|_{2}^{4}+\left\|u^{m}\right\|_{2}\right) .
\end{aligned}
$$

Combining 3.12 and 3.13 to have

$$
\begin{gathered}
\int_{\Omega}\left|u_{t}^{m}\right|^{\rho}\left|u_{t t}^{m}\right|^{2} d x+(1-c \delta)\left\|\Delta u_{t t}^{m}\right\|_{2}^{2} \leq \frac{1}{4 \delta}\left(\int_{0}^{t} g(t-s)\left\|\Delta u^{m}(s)\right\|_{2} d s\right)^{2} \\
+\frac{1}{4 \delta}\left\|\Delta u^{m}\right\|^{2}+\frac{c}{\delta}\left(\left\|\Delta u^{m}\right\|_{2}^{4}+\left\|u^{m}\right\|_{2}^{2}\right) .
\end{gathered}
$$

Integrate the last inequality on $(0, T)$ and use 2.1 and (3.11), we obtain

$$
\begin{aligned}
& \int_{0}^{T} \int_{\Omega}\left|u_{t}^{m}\right|^{\rho}\left|u_{t t}^{m}\right|^{2} d x d t+(1-c \delta) \int_{0}^{T}\left\|\Delta u_{t t}^{m}\right\|_{2}^{2} d t \\
& \leq \frac{c}{\delta} \int_{0}^{T}\left[\left(g o \Delta u^{m}\right)(t)+\left\|\Delta u^{m}\right\|_{2}^{2}+\left\|\Delta u^{m}\right\|_{2}^{4}+\left\|u^{m}\right\|_{2}^{2}\right] d t
\end{aligned}
$$

From the last inequality, choosing $\delta>0$ small enough and using (3.11), we get the following, for some positive constant $C_{4}$ not depending neither on $m$ nor on $t$ :

$$
\int_{0}^{T}\left\|\Delta u_{t t}^{m}\right\|_{2}^{2} d t \leq C_{4} .
$$

From 3.11 and 3.16 , we have

$$
\left\{\begin{array}{l}
\left(u^{m}\right) \text { is uniformly bounded in } L^{\infty}\left(0, T ; H_{0}^{2}(\Omega)\right), \\
\left(u_{t}^{m}\right) \text { is uniformly bounded in } L^{\infty}\left(0, T ; L^{\rho+2}(\Omega)\right) \cap L^{\infty}\left(0, T ; H_{0}^{2}(\Omega)\right), \\
\left(u_{t t}^{m}\right) \text { is uniformly bounded in } L^{2}\left(0, T ; H_{0}^{2}(\Omega)\right),
\end{array}\right.
$$


which implies that there exists a subsequence of $\left(u^{m}\right)$ (still denoted by $\left(u^{m}\right)$ ), such that

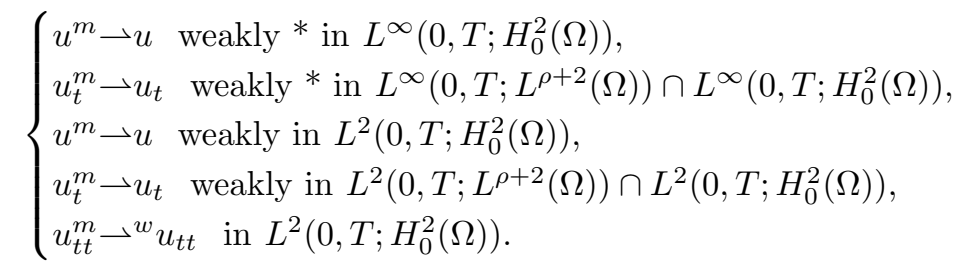

\section{Analysis of the non-linear terms}

(1) Term $u^{m} \ln \left|u^{m}\right|$ : using (3.17), we have $\left(u^{m}\right)$ is bounded in $L^{\infty}\left(0, T ; H_{0}^{2}(\Omega)\right)$ which implies, using the embedding of $H_{0}^{2}(\Omega)$ in $L^{\infty}(\Omega)\left(\Omega \subset \mathbb{R}^{2}\right)$, the boundedness of $\left(u^{m}\right)$ in $L^{2}(\Omega \times(0, T))$. Similarly; $\left(u_{t}^{m}\right)$ is bounded in $L^{2}(\Omega \times(0, T))$. Then, making use of Aubin-Lions' theorem, we find, up to a subsequence, that

$$
u^{m} \rightarrow u \text { strongly in } L^{2}(\Omega \times(0, T))
$$

and

$$
u^{m} \rightarrow u \text { a.e. in } \Omega \times(0, T) .
$$

Since the maps $s \rightarrow k s \ln |s|$ is continuous, we have the following convergence:

$$
k u^{m} \ln \left|u^{m}\right| \rightarrow k u \ln |u| \text { a.e. in } \Omega \times(0, T) .
$$

Using the embedding of $H_{0}^{2}(\Omega)$ in $L^{\infty}(\Omega)\left(\Omega \subset \mathbb{R}^{2}\right)$, it is clear that $k\left(u^{m} \ln \left|u^{m}\right|\right)$ is bounded in $L^{\infty}(\Omega \times(0, T))$. Next, taking into account the Lebesgue bounded convergence theorem ( $\Omega$ is bounded), we get

$$
k u^{m} \ln \left|u^{m}\right| \rightarrow k u \ln |u| \text { strongly in } L^{2}\left(0, T ; L^{2}(\Omega)\right) .
$$

(2) Term $\left|u_{t}^{m}\right|^{\rho} u_{t}^{m}$ : using (3.16), we have $\left(u_{t}^{m}\right)$ is uniformly bounded in $L^{\infty}\left(0, T ; H_{0}^{2}(\Omega)\right)$ which implies the boundedness of $\left(u_{t}^{m}\right)$ in $L^{\infty}(\Omega \times(0, T))$, and so in $L^{2}(\Omega \times$ $(0, T))$. Using (3.16), we see that $\left(u_{t t}^{m}\right)$ is bounded in $L^{2}\left((0, T) ; H_{0}^{2}(\Omega)\right)$ which implies that $\left(u_{t t}^{m}\right)$ is bounded in $L^{2}(\Omega \times(0, T))$. Now, using AubinLions theorem, there exists a subsequence, still denoted by $\left(u_{t}^{m}\right)$, such that

$$
u_{t}^{m} \rightarrow u_{t} \text { strongly in } L^{2}\left(0, T ; L^{2}(\Omega)\right)
$$

and

$$
\left|u_{t}^{m}\right|^{\rho} u_{t}^{m} \rightarrow\left|u_{t}\right|^{\rho} u_{t} \text { a.e. in } \Omega \times(0, T) .
$$

Using (3.11) and the embedding theorems, we have

$$
\begin{aligned}
\left\|\left|u_{t}^{m}\right|^{\rho} u_{t}\right\|_{L^{2}\left(0, T ; L^{2}(\Omega)\right)}^{2} & =\int_{0}^{T}\left\|u_{t}^{m}\right\|_{2(\rho+1)}^{2(\rho+1)} d t \\
& \leq c \int_{0}^{T}\left\|\Delta u_{t}^{m}(t)\right\|_{2}^{2(\rho+1)} d t \leq c T C_{3}^{\rho+1},
\end{aligned}
$$

which implies that $\left(\left|u_{t}^{m}\right|^{\rho} u_{t}^{m}\right)$ is bounded in $L^{2}(\Omega \times(0, T))$. Combining (3.21) and 3.22 and using Lions' lemma, see Lions (26], pp. 12), we obtain

$$
\left|u_{t}^{m}\right|^{\rho} u_{t}^{m}-\left|u_{t}\right|^{\rho} u_{t} \text { weakly in } L^{2}\left(0, T ; L^{2}(\Omega)\right) .
$$


Now, we integrate 3.3 over $(0, t)$ to obtain, for every $w \in V_{m}$,

$$
\begin{gathered}
\frac{1}{\rho+1} \int_{\Omega}\left|u_{t}^{m}\right|^{\rho} u_{t}^{m} w d x d s-\frac{1}{\rho+1} \int_{\Omega}\left|u_{1}^{m}\right| u_{1}^{m} w d x+\int_{0}^{t} \int_{\Omega} \Delta u^{m}(s) \Delta w d x d s \\
+\int_{\Omega} \Delta u_{t}^{m} \Delta w d x d s-\int_{\Omega} \Delta u_{1}^{m} \Delta w d x-\int_{\Omega} \int_{0}^{t}\left(\int_{0}^{\tau} g(\tau-s) \Delta u^{m}(s)\right) \Delta w d s d \tau d x \\
=k \int_{0}^{t} \int_{\Omega} w u^{m}(s) \ln \left|u^{m}(s)\right| d x d s .
\end{gathered}
$$

Convergences 3.2), 3.18, 3.20 and 3.23 are sufficient to pass to the limit in (3.24) as $m \rightarrow \infty$, and get, for any $w \in V_{m}$ and $m \geq 1$,

$$
\begin{aligned}
& \frac{1}{\rho+1} \int_{0}^{t} \int_{\Omega}\left|u_{s}\right|^{\rho} u_{s} w d x d s=\frac{1}{\rho+1} \int_{\Omega}\left|u_{1}^{m}\right| u_{1}^{m} w d x-\int_{0}^{t} \int_{\Omega} \Delta u(s) \Delta w d x d s \\
& \quad-\int_{\Omega} \Delta u_{t} \Delta w d x d s+\int_{\Omega} \Delta u_{1}^{m} \Delta w d x+\int_{\Omega} \int_{0}^{t}\left(\int_{0}^{\tau} g(\tau-s) \Delta u(s)\right) \Delta w d s d \tau d x \\
& \quad+k \int_{0}^{t} \int_{\Omega} w u(s) \ln |u(s)| d x d s,
\end{aligned}
$$

which implies that 3.25 is valid for any $w \in H_{0}^{2}(\Omega)$. Using the fact that the terms in the right-hand side of $(3.25$ are absolutely continuous (since they are functions of $t$ defined by integrals over $(0, t))$, then $\left(3.25\right.$ is differentiable for a.e. $t \in \mathbb{R}^{+}$. Thus, differentiating 3.25 , we obtain, for a.e. $t \in(0, T)$ and $w \in H_{0}^{2}(\Omega)$,

$$
\begin{aligned}
& \int_{\Omega}\left|u_{t}\right|^{\rho} u_{t t} w d x d s+\int_{\Omega} \Delta u(t) \Delta w d x \\
& +\int_{\Omega} \Delta u_{t t} \Delta w d x-\int_{\Omega}\left(\int_{0}^{t} g(t-s) \Delta u(s)\right) \Delta w d s d x \\
& \quad=k \int_{\Omega} w u(t) \ln |u(t)| d x d s .
\end{aligned}
$$

This ends the proof of Theorem 3.2 .

\section{Global Existence}

In this section, we state and prove a global existence result under smallness conditions on the initial data $\left(u_{0}, u_{1}\right)$. For this purpose, we introduce the following functionals:

$$
\begin{gathered}
J(t)=\frac{1}{2}\left(\left(1-\int_{0}^{t} g(s) d s\right)\|\Delta u\|_{2}^{2}+\left\|\Delta u_{t}\right\|_{2}^{2}+g o \Delta u-k \int_{\Omega} u^{2} \ln |u| d x\right) \\
+\frac{k}{4}\|u\|_{2}^{2}
\end{gathered}
$$

and

$$
I(t)=\left(1-\int_{0}^{t} g(s) d s\right)\|\Delta u\|_{2}^{2}+\left\|\Delta u_{t}\right\|_{2}^{2}+g o \Delta u-3 k \int_{\Omega} u^{2} \ln |u| d x .
$$


Lemma 4.1. The following inequalities hold:

$$
-k d_{0} \sqrt{|\Omega| c_{*}^{3}}\left\|\left.\Delta u\right|_{2} ^{\frac{3}{2}} \leq k \int_{\Omega} u^{2} \ln |u| d x \leq k c_{*}^{3}\right\| \Delta u \|_{2}^{3}, \quad \forall u \in H_{0}^{2}(\Omega),
$$

where $d_{0}=\sup _{0<s<1} \sqrt{s}|\ln s|=\frac{2}{e},|\Omega|$ is the Lesbegue measure of $\Omega$ and $c_{*}$ is the smallest embedding constant

$$
\left(\int_{\Omega}|u|^{3} d x\right)^{\frac{1}{3}} \leq c_{*}\|\Delta u\|_{2}, \quad \forall u \in H_{0}^{2}(\Omega)
$$

( $c_{*}$ exists thanks to the embedding of $H_{0}^{2}(\Omega)$ in $L^{\infty}(\Omega)$ and $\Omega \subset \mathbb{R}^{2}$ ).

Proof. Let

$$
\Omega_{1}=\{x \in \Omega:|u(x)|>1\} \text { and } \Omega_{2}=\{x \in \Omega:|u(x)| \leq 1\} .
$$

So, using (4.4), we have

$$
\begin{aligned}
& k \int_{\Omega} u^{2} \ln |u| d x=k \int_{\Omega_{2}} u^{2} \ln |u| d x+k \int_{\Omega_{1}} u^{2} \ln |u| d x \\
& \quad \leq k \int_{\Omega_{1}} u^{2} \ln |u| d x \leq k \int_{\Omega_{1}}|u|^{3} d x \leq k \int_{\Omega}|u|^{3} d x \leq k c_{*}^{3}\|\Delta u\|_{2}^{3} .
\end{aligned}
$$

On the other hand, using Hölder's inequality and 4.4, we find

$$
\begin{aligned}
& -k \int_{\Omega} u^{2} \ln |u| d x=-k \int_{\Omega_{2}} u^{2} \ln |u| d x-k \int_{\Omega_{1}} u^{2} \ln |u| d x \\
& \quad \leq-k \int_{\Omega_{2}} u^{2} \ln |u| d x=k \int_{\Omega_{2}} u^{2}|\ln | u|| d x \\
& \leq k d_{0} \int_{\Omega}|u|^{\frac{3}{2}} d x \leq k d_{0} \sqrt{|\Omega|}\left(\int_{\Omega}|u|^{3} d x\right)^{\frac{1}{2}} \leq k d_{0} \sqrt{|\Omega| c_{*}^{3}}\|\Delta u\|_{2}^{\frac{3}{2}},
\end{aligned}
$$

which implies the left inequality in 4.3 .

Lemma 4.2. Assume that $(A 1)-(A 3)$. Let $\left(u_{0}, u_{1}\right) \in H_{0}^{2}(\Omega) \times H_{0}^{2}(\Omega)$ such that

$$
I(0)>0 \text { and } \sqrt{54} k c_{*}^{3}\left(\frac{E(0)}{\ell}\right)^{\frac{1}{2}}<\ell .
$$

Then

$$
I(t)>0, \forall t \in[0, T) .
$$

Proof. From 4.2, we have

$$
k \int_{\Omega} u^{2} \ln |u| d x=\frac{1}{3}\left(1-\int_{0}^{t} g(s) d s\right)\|\Delta u\|_{2}^{2}+\frac{1}{3}\left\|\Delta u_{t}\right\|_{2}^{2}+\frac{1}{3} g o \Delta u-\frac{1}{3} I(t) .
$$

Substitute (4.7) in 4.1), we find

$$
J(t)=\frac{1}{3}\left[\left(1-\int_{0}^{t} g(s) d s\right)\|\Delta u\|_{2}^{2}+\left\|\Delta u_{t}\right\|_{2}^{2}+g o \Delta u\right]+\frac{k}{4}\|u\|_{2}^{2}+\frac{1}{6} I(t) .
$$

Since $I(0)>0$ and $I$ is continuous on $[0, T]$, there exists $t_{0} \in(0, T]$ such that $I(t)>0$, for all $t \in\left[0, t_{0}\right)$. Let us denote by $t_{0}$ the largest real number in $(0, T]$ such that $I>0$ on $\left[0, t_{0}\right)$. If $t_{0}=T$, then (4.6) is satisfied.

We assume by contradiction that $t_{0} \in(0, T)$. Thus $I\left(t_{0}\right)=0$ and

$$
\|\Delta u(t)\|_{2}^{2} \leq \frac{6}{\ell} J(t) \leq \frac{6}{\ell} E(t) \leq \frac{6}{\ell} E(0), \forall t \in\left[0, t_{0}\right) .
$$


If $\left\|\Delta u\left(t_{0}\right)\right\|_{2}^{2}=0$, then 4.3 and 4.4 give

$$
\begin{aligned}
0=I\left(t_{0}\right)= & \left(1-\int_{0}^{t_{0}} g(s) d s\right)\left\|\Delta u\left(t_{0}\right)\right\|_{2}^{2}+\left\|\Delta u_{t}\left(t_{0}\right)\right\|_{2}^{2}+g o \Delta u\left(t_{0}\right) \\
& \quad-3 k \int_{\Omega} u^{2}\left(t_{0}\right) \ln \left|u\left(t_{0}\right)\right| d x \\
\leq & c\left\|\Delta u\left(t_{0}\right)\right\|_{2}^{2}+g o \Delta u\left(t_{0}\right) \\
= & \int_{0}^{t_{0}} g(s)\|\Delta u(s)\|_{2}^{2} d s .
\end{aligned}
$$

Consequently, if $g>0$ on $\left[0, t_{0}\right)$, we get

$$
\|\Delta u(s)\|_{2}=0, \quad \forall s \in\left[0, t_{0}\right) .
$$

Then

$$
I(t)=0, \quad \forall t \in\left[0, t_{0}\right),
$$

which is not true since $I>0$ on $\left[0, t_{0}\right)$. If $g \neq 0$ on $\left[0, t_{0}\right)$, then let $t_{1} \in\left[0, t_{0}\right)$ the smallest real number such that $g\left(t_{1}\right)=0$. Because $g(0)>0$ and $g$ is positive, nonincreasing and continuous on $\mathbb{R}^{+}$(condition $(A 1)$ ), then $t_{1}>0$ and $g=0$ on $\left[t_{1}, \infty\right)$. Therefore, from 4.10 , we deduce that

$$
0=\int_{0}^{t_{0}} g(s)\|\Delta u(s)\|_{2}^{2} d s=\int_{0}^{t_{1}} g(s)\|\Delta u(s)\|_{2}^{2} d s,
$$

then $\|\Delta u(s)\|_{2}=0$, for any $s \in\left[0, t_{1}\right)$, which implies that $I(t)=0$, for any $t \in\left[0, t_{1}\right)$. As in above, this is a contraduction with the fact that $I>0$ on $\left[0, t_{0}\right)$. Then we conclude that $\left\|\Delta u\left(t_{0}\right)\right\|_{2}^{2}>0$. On the other hannd, we have

$$
I\left(t_{0}\right) \geq \ell\left\|\Delta u\left(t_{0}\right)\right\|_{2}^{2}-3 k \int_{\Omega} u\left(t_{0}\right)^{2} \ln \left|u\left(t_{0}\right)\right| d x .
$$

By using 4.9 and Lemma 4.1, we have

$$
I\left(t_{0}\right) \geq\left[\ell-3 k c_{*}^{3}\left(\frac{6 E(0)}{\ell}\right)^{\frac{1}{2}}\right]\left\|\Delta u\left(t_{0}\right)\right\|_{2}^{2} .
$$

By recalling (4.5), we arrive at $I\left(t_{0}\right)>0$, which contradicts the assumption $I\left(t_{0}\right)=$ 0 . Hence, $t_{0}=T$ and then

$$
I(t)>0, \forall t \in[0, T) .
$$

\section{Stability}

In this section, we state and prove our stability result. We start by establishing several lemmas needed for the proof of our main result.

Lemma 5.1. Assume that $g$ satisfies $(A 1)$. Then, for $u \in H_{0}^{2}(\Omega)$, we have

$$
\int_{\Omega}\left(\int_{0}^{t} g(t-s)(u(t)-u(s)) d s\right)^{2} d x \leq c(g \circ \Delta u)(t)
$$

and

$$
\int_{\Omega}\left(\int_{0}^{t} g^{\prime}(t-s)(u(t)-u(s)) d s\right)^{2} d x \leq-c\left(g^{\prime} o \Delta u\right)(t) .
$$


Proof.

$\int_{\Omega}\left(\int_{0}^{t} g(t-s)(u(t)-u(s)) d s\right)^{2} d x=\int_{\Omega}\left(\int_{0}^{t} \sqrt{g(t-s)} \sqrt{g(t-s)}(u(t)-u(s)) d s\right)^{2} d x$.

By applying Cauchy-Schwarz' and Poincaré's inequalities, we can show that

$$
\begin{aligned}
& \int_{\Omega}\left(\int_{0}^{t} g(t-s)(u(t)-u(s)) d s\right)^{2} d x \\
& \quad \leq \int_{\Omega}\left(\int_{0}^{t} g(t-s) d s\right)\left(\int_{0}^{t} g(t-s)(u(t)-u(s))^{2} d s\right) d x \\
& \quad \leq(1-\ell) c(g o \Delta u)(t) \\
& \quad \leq c(g o \Delta u)(t) .
\end{aligned}
$$

Similarly, the second inequality in Lemma 5.1 can be proved.

Lemma 5.2. Assume that $g$ satisfies $(A 1)$ and $(A 2)$. Then

$$
\int_{0}^{\infty} \xi(t) g^{1-\sigma}(t) d t<\infty, \quad \forall \sigma<2-p
$$

Proof. Using (A1) and (A2), we easily see that, for any $\sigma<2-p$,

$$
\xi(t) g^{1-\sigma}(t)=\xi(t) g^{1-\sigma}(t) g^{p}(t) g^{-p}(t) \leq-g^{\prime}(t) g^{1-\sigma-p}(t) .
$$

Integrate the last inequality over $(0, \infty)$, we obtain

$$
\int_{0}^{\infty} \xi(t) g^{1-\sigma}(t) d t \leq-\int_{0}^{\infty} g^{\prime}(t) g^{1-\sigma-p}(t) d t=\left[-\frac{g^{2-p-\sigma}(t)}{2-p-\sigma}\right]_{0}^{\infty}<\infty .
$$

Similar to Cavalcanti and Oquendo [7, we can easily have the following lemma:

Lemma 5.3. Assume that $(A 1)-(A 3)$ and 4.5 hold and $u$ is a solution of (1.1). Then, for any $0<\sigma<1$, we have

$$
(g \circ \Delta u)(t) \leq c\left[\left(\int_{0}^{t} g^{1-\sigma}(t) d t\right) E(0)\right]^{\frac{p-1}{p-1+\sigma}}\left(g^{p} o \Delta u\right)^{\frac{\sigma}{p-1+\sigma}} .
$$

By taking $\sigma=\frac{1}{2}$, we get

$$
(g o \Delta u)(t) \leq c\left(\int_{0}^{t} g^{\frac{1}{2}}(s) d s\right)^{\frac{2 p-2}{2 p-1}}\left(g^{p} o \Delta u\right)^{\frac{1}{2 p-1}}(t)
$$

and, for any $\epsilon_{0} \in(0,1)$,

$$
(g \circ \Delta u)^{\frac{1}{1+\epsilon_{0}}}(t) \leq c^{\frac{1}{1+\epsilon_{0}}}\left(\int_{0}^{t} g^{\frac{1}{2}}(s) d s\right)^{\frac{2 p-2}{(2 p-1)\left(1+\epsilon_{0}\right)}}\left(g^{p} o \Delta u\right)^{\frac{1}{(2 p-1)\left(1+\epsilon_{0}\right)}}(t) .
$$

Corollary 5.4. Assume that $(A 1)-(A 3)$ and 4.5 hold and $u$ is a solution of (1.1. Then

$$
\xi(t)(g o \Delta u)(t) \leq c\left(-E^{\prime}(t)\right)^{\frac{1}{2 p-1}}
$$

and, for any $\epsilon_{0} \in(0,1)$,

$$
\xi(t)(g o \Delta u)^{\frac{1}{1+\epsilon_{0}}}(t) \leq c_{\epsilon_{0}}\left(-E^{\prime}(t)\right)^{\frac{1}{(2 p-1)\left(1+\epsilon_{0}\right)}} .
$$


Proof. Multiply both sides of 5.3 by $\xi(t)$ and use 5.2 and 2.5 to obtain

$$
\begin{aligned}
\xi(t)(g o \Delta u)(t) & \leq c \xi^{\frac{2 p-2}{2 p-1}}(t)\left(\int_{0}^{t} g^{\frac{1}{2}}(s) d s\right)^{\frac{2 p-2}{22-1}} \xi^{\frac{1}{2 p-1}}(t)\left(g^{p} o \Delta u\right)^{\frac{1}{2 p-1}}(t) \\
\leq & c\left(\int_{0}^{t} \xi(s) g^{\frac{1}{2}}(s) d s\right)^{\frac{2 p-2}{2 p-1}}\left(\xi g^{p} o \Delta u\right)^{\frac{1}{2 p-1}}(t) \\
\leq & c\left(\int_{0}^{\infty} \xi(s) g^{\frac{1}{2}}(s) d s\right)^{\frac{2 p-2}{2 p-1}}\left(-g^{\prime} o \Delta u\right)^{\frac{1}{2 p-1}}(t) \\
& \leq c\left(-E^{\prime}(t)\right)^{\frac{1}{2 p-1}} .
\end{aligned}
$$

For the proof of (5.6), using (5.5) and because $\xi$ is nonnegative and nonincreasing, we obtain

$$
\xi(t)(g \circ \Delta u)^{\frac{1}{1+\epsilon_{0}}}(t)=\xi^{\frac{\epsilon_{0}}{1+\epsilon_{0}}}(t)(\xi(t)(g o \Delta u)(t))^{\frac{1}{1+\epsilon_{0}}} \leq c_{\epsilon_{0}}\left(-E^{\prime}(t)\right)^{\frac{1}{(2 p-1)\left(1+\epsilon_{0}\right)}} .
$$

Lemma 5.5. Under the assumptions $(A 1)-(A 3)$ and $[4.5)$, the functional

$$
\psi(t):=\frac{1}{\rho+1} \int_{\Omega}\left|u_{t}\right|^{\rho} u_{t} u d x+\int_{\Omega} \Delta u \Delta u_{t} d x
$$

satisfies, along the solution of (1.1), the estimate

$$
\begin{aligned}
\psi^{\prime}(t) \leq- & \frac{\ell}{2} \int_{\Omega}|\Delta u|^{2} d x+\int_{\Omega}\left|\Delta u_{t}\right|^{2} d x+\frac{1}{\rho+1} \int_{\Omega}\left|u_{t}\right|^{\rho+2} d x+c(g o \Delta u)(t) \\
& +k \int_{\Omega} u^{2} \ln |u| d x
\end{aligned}
$$

Proof. Direct differentiation of $\psi$, using (1.1), yields

$$
\begin{aligned}
\psi^{\prime}(t)= & -\int_{\Omega}|\Delta u|^{2} d x+\int_{\Omega} \Delta u(t) \int_{0}^{t} g(t-s) \Delta u(s) d s d x \\
& +\int_{\Omega}\left|\Delta u_{t}\right|^{2} d x+\frac{1}{\rho+1} \int_{\Omega}\left|u_{t}\right|^{\rho+2} d x+k \int_{\Omega} u^{2} \ln |u| d x .
\end{aligned}
$$

We then estimate the second term on the right side of (5.9). We have, using (2.1),

$$
\begin{gathered}
\int_{\Omega} \Delta u(t) \int_{0}^{t} g(t-s) \Delta u(s) d s d x=\int_{\Omega} \Delta u(t) \int_{0}^{t} g(t-s)(\Delta u(s)-\Delta u(t)+\Delta u(t)) d s d x \\
\leq(1-\ell) \int_{\Omega}|\Delta u|^{2} d x-\int_{\Omega}\left(\int_{0}^{t} g(t-s)(\Delta u(t)-\Delta u(s)) d s\right) d x .
\end{gathered}
$$

By exploiting Lemma 5.1 and

$$
a b \leq \frac{1}{2 \eta} a^{2}+\frac{\eta}{2} b^{2}, \quad \forall a, b \geq 0, \forall \eta>0,
$$


we arrive at

$$
\begin{aligned}
& \int_{\Omega} \Delta u(t) \int_{0}^{t} g(t-s) \Delta u(s) d s d x \\
& \leq(1-\ell) \int_{\Omega}|\Delta u|^{2} d x+\frac{1}{2 \eta} \int_{\Omega}\left(\int_{0}^{t} g(t-s)|\Delta u(t)-\Delta u(s)| d s\right)^{2} d x \\
& \quad+\frac{\eta}{2} \int_{\Omega}|\Delta u|^{2} d x \\
& \leq\left(1-\ell+\frac{\eta}{2}\right) \int_{\Omega}|\Delta u|^{2} d x+\frac{c}{\eta}(g o \Delta u)(t)
\end{aligned}
$$

By taking $\eta=\ell$, we find

$$
\int_{\Omega} \Delta u(t) \int_{0}^{t} g(t-s) \Delta u(s) d s d x \leq \frac{2-\ell}{2} \int_{\Omega}|\Delta u|^{2} d x+c(g o \Delta u)(t) .
$$

Inserting 5.10 in 5.9$)$, estimate $(5.8)$ is established.

Lemma 5.6. Under the assumptions $(A 1)-(A 3)$ and 4.5 , the functional

$$
\chi(t):=-\int_{\Omega}\left(\Delta^{2} u_{t}+\frac{1}{\rho+1}\left|u_{t}\right|^{\rho} u_{t}\right) \int_{0}^{t} g(t-s)(u(t)-u(s)) d s d x
$$

satisfies, along the solution of (1.1) and for any $\delta, \delta_{1}, \delta_{2}>0$, the estimate

$$
\begin{aligned}
\chi^{\prime}(t) & \leq\left[\left(1+2(1-\ell)^{2}\right) \delta_{1}+\frac{\delta}{4}\right] \int_{\Omega}|\Delta u|^{2} d x-\frac{1}{\rho+1}\left(\int_{0}^{t} g(s) d s\right) \int_{\Omega}\left|u_{t}\right|^{\rho+2} d x \\
& +c\left(\delta_{1}+\frac{1}{\delta_{1}}+\frac{1}{\delta}\right)(g o \Delta u)(t)-\frac{c}{\delta_{2}}\left(g^{\prime} o \nabla u\right)(t) \\
& +\left[\delta_{2}+c \delta_{2}(E(0))^{\rho}-\int_{0}^{t} g(s) d s\right] \int_{\Omega}\left|\Delta u_{t}\right|^{2} d x+c_{\epsilon_{0}, \delta}(g o \Delta u)^{\frac{1}{1+\epsilon_{0}}}(t) .
\end{aligned}
$$

Proof. Differentiating $\chi$ with respect to $t$ and making use of 1.1 , we find

$$
\begin{aligned}
\chi^{\prime}(t) & =\int_{\Omega} \Delta u(t) \int_{0}^{t} g(t-s)(\Delta u(s)-\Delta u(t)) d s d x \\
& -\int_{\Omega}\left(\int_{0}^{t} g(t-s) \Delta u(s) d s\right)\left(\int_{0}^{t} g(t-s)(\Delta u(s)-\Delta u(t)) d s\right) d x \\
& -\left(\int_{0}^{t} g(s) d s\right) \int_{\Omega}\left|\Delta u_{t}\right|^{2} d x-\int_{\Omega} \Delta u_{t}(t) \int_{0}^{t} g^{\prime}(s)(\Delta u(s)-\Delta u(t)) d s d x \\
& -\frac{1}{\rho+1} \int_{\Omega}\left|u_{t}\right|^{\rho} u_{t} \int_{0}^{t} g^{\prime}(t-s)(u(s)-u(t)) d s d x \\
& -\frac{1}{\rho+1}\left(\int_{0}^{t} g(s) d s\right) \int_{\Omega}\left|u_{t}\right|^{\rho+2} d x-k \int_{\Omega} u \ln |u| \int_{0}^{t} g(t-s)(u(t)-u(s)) d s d x .
\end{aligned}
$$

Now we proceed, using repeatedly Cauchy-Schwarz' inequality, Young's inequality and Lemma 5.1. to estimate each term in the right-hand side of 5.12. The first 
term may be estimated as follows

$$
\begin{aligned}
\int_{\Omega} \Delta & u(t) \int_{0}^{t} g(t-s)(\Delta u(s)-\Delta u(t)) d s d x \\
& \leq \delta_{1} \int_{\Omega}|\Delta u|^{2} d x+\frac{c}{\delta_{1}}(g o \Delta u)(t), \quad \forall \delta_{1}>0 .
\end{aligned}
$$

For the second term, we recall (2.1) and the fact that $(a+b)^{2} \leq 2\left(a^{2}+b^{2}\right)$ to get, for any $\delta_{1}>0$,

$$
\begin{aligned}
& -\int_{\Omega}\left(\int_{0}^{t} g(t-s) \Delta u(s) d s\right)\left(\int_{0}^{t} g(t-s)(\Delta u(s)-\Delta u(t)) d s\right) d x \\
& \quad \leq \delta_{1} \int_{\Omega}\left|\int_{0}^{t} g(t-s) \Delta u(s) d s\right|^{2} d x+\frac{1}{4 \delta_{1}} \int_{\Omega}\left|\int_{0}^{t} g(t-s)(\Delta u(s)-\Delta u(t)) d s\right|^{2} d x \\
& \quad \leq \delta_{1} \int_{\Omega}\left(\int_{0}^{t} g(t-s)(|\Delta u(s)-\Delta u(t)|+|\Delta u(t)|) d s\right)^{2} d x+\frac{c}{\delta_{1}}(g o \Delta u)(t) \\
& \leq c\left(\delta_{1}+\frac{1}{\delta_{1}}\right)(g o \Delta u)(t)+2 \delta_{1}(1-\ell)^{2} \int_{\Omega}|\Delta u|^{2} d x .
\end{aligned}
$$

For the fourth term, it is easy to see that, for any $\delta_{2}>0$,

$$
\begin{aligned}
& -\int_{\Omega} \Delta u_{t} \int_{0}^{t} g^{\prime}(t-s)(\Delta u(s)-\Delta u(t)) d s d x \\
& \quad \leq \delta_{2} \int_{\Omega}\left|\Delta u_{t}\right|^{2} d x+\frac{c}{\delta_{2}} \int_{\Omega} \int_{0}^{t}\left(-g^{\prime}(t-s)\right)|\Delta u(s)-\Delta u(t)|^{2} d s d x .
\end{aligned}
$$

The fifth term may be handled similarly

$$
\begin{aligned}
& -\frac{1}{\rho+1} \int_{\Omega}\left|u_{t}\right|^{\rho} u_{t} \int_{0}^{t} g^{\prime}(t-s)(u(s)-u(t)) d s d x \\
& \quad \leq \frac{1}{\rho+1}\left[\delta_{2} \int_{\Omega}\left|u_{t}\right|^{2(\rho+1)} d x+\frac{c}{\delta_{2}} \int_{\Omega} \int_{0}^{t}\left(-g^{\prime}(t-s)\right)|\Delta u(s)-\Delta u(t)|^{2} d s d x\right] .
\end{aligned}
$$

Using (2.4), 2.5), 4.1), 4.6 and 4.8, we have

$$
E(0) \geq E(t)=J(t)+\frac{1}{\rho+2}\left\|u_{t}\right\|_{\rho+2}^{\rho+2} \geq J(t) \geq \frac{1}{6}\left\|\Delta u_{t}\right\|_{2}^{2},
$$

which gives

$$
\left\|\Delta u_{t}\right\|_{2}^{2} \leq 6 E(0) .
$$

By exploiting the Sobolev embedding

$$
H_{0}^{1}(\Omega) \hookrightarrow L^{2(\rho+1)}(\Omega)
$$

and (5.17), we obtain

$$
\int_{\Omega}\left|u_{t}\right|^{2(\rho+1)} d x \leq c(E(0))^{\rho}\left\|\Delta u_{t}\right\|_{2}^{2}
$$


Therefore 5.16 takes the form

$$
\begin{gathered}
-\frac{1}{\rho+1} \int_{\Omega}\left|u_{t}\right|^{\rho} u_{t} \int_{0}^{+\infty} g^{\prime}(s)(u(t-s)-u(t)) d s d x \\
\leq c \delta_{2}(E(0))^{\rho}\left\|\Delta u_{t}\right\|_{2}^{2}-\frac{c}{\delta_{2}}\left(g^{\prime} o \Delta u\right)(t)
\end{gathered}
$$

Applying 2.10 for $s=|u|$, using the embedding of $H_{0}^{2}(\Omega)$ in $L^{\infty}(\Omega)$ and performing the same calulactions as before, we get, for any $\delta_{3}>0$ and any $\varepsilon_{0} \in(0,1)$,

$$
\begin{aligned}
& -k \int_{\Omega} u \ln |u| \int_{0}^{t} g(t-s)(u(t)-u(s)) d s d x \\
& \leq k \int_{\Omega}\left(u^{2}+d_{\epsilon_{0}}|u|^{1-\epsilon_{0}}\right)\left|\int_{0}^{t} g(t-s)(u(t)-u(s)) d s d x\right| \\
& \leq c \int_{\Omega}|u|^{2}\left|\int_{0}^{t} g(t-s)(u(t)-u(s)) d s\right| d x+\delta_{3} \int_{\Omega} u^{2} d x \\
& \quad+c_{\epsilon_{0}, \delta_{3}} \int_{\Omega}\left|\int_{0}^{t} g(t-s)(u(t)-u(s)) d s\right|^{\frac{2}{1+\epsilon_{0}}} d x \\
& \leq c \delta_{3}|| \Delta u \|_{2}^{2}+\frac{c}{\delta_{3}} \int_{\Omega}\left|\int_{0}^{t} g(t-s)(u(t)-u(s)) d s\right|^{2} d x \\
& \quad+c_{\epsilon_{0}, \delta_{3}} \int_{\Omega}\left|\int_{0}^{t} g(t-s)(u(t)-u(s)) d s\right|^{\frac{2}{1+\epsilon_{0}}} d x
\end{aligned}
$$

then, puting $\frac{\delta}{4}=c \delta_{3}$ and using Holder's inequality and Lemma 5.1 , we find

$$
\begin{aligned}
-k \int_{\Omega} u \ln |u| \int_{0}^{t} g(t-s)(u(t)-u(s)) d s d x & \leq \frac{\delta}{4}\|\Delta u\|_{2}^{2}+\frac{c}{\delta}(g o \Delta u)(t) \\
& +c_{\epsilon_{0}, \delta}(g o \Delta u)^{\frac{1}{1+\epsilon_{0}}}(t) .
\end{aligned}
$$

Combining (5.12)-5.15), 5.20 and 5.21), estimate (5.11) is established.

Lemma 5.7. Assume that $(A 1)-(A 3)$ and 4.5 hold and let $\varepsilon_{0} \in(0,1)$. Assume that

$$
0<E(0)<\frac{e \ell \pi}{4 c_{p}} .
$$

Then, for $k$ small enough, there exist positive constants $\varepsilon$ and $N$ such that the functional

$$
L=N E+\varepsilon \psi+\chi
$$

satisfies

$$
L \sim E
$$

and, for any $t_{0}>0$, there exists a positive constant $m$ such that

$$
L^{\prime}(t) \leq-m E(t)+c(g o \Delta u)(t)+c_{\epsilon_{0}}(g o \Delta u)^{\frac{1}{1+\epsilon_{0}}}(t), \quad \forall t \geq t_{0} .
$$


Proof. To prove (5.23), we use Young's inequality, the Sobolev embedding $H_{0}^{1}(\Omega) \hookrightarrow$ $L^{\rho+2}(\Omega), 4.6$, (4.8), (5.17) and 5.19) to obtain

$$
\begin{aligned}
|L(t)-N E(t)| \leq \frac{\varepsilon}{\rho+2} & \left\|u_{t}\right\|_{\rho+2}^{\rho+2}+\frac{\varepsilon}{(\rho+1)(\rho+2)}\|u\|_{\rho+2}^{\rho+2}+\frac{\varepsilon}{2}\left\|\Delta u_{t}\right\|_{2}^{2} \\
& +\frac{\varepsilon}{2}\|\Delta u\|_{2}^{2}+\frac{1}{2(\rho+1)}\left\|u_{t}\right\|_{2(\rho+1)}^{2(\rho+1)}+\frac{1-\ell}{2(\rho+1)} c_{p}(g o \Delta u)(t) \\
& +\frac{1}{2}\left\|\Delta u_{t}\right\|_{2}^{2}+\frac{1-\ell}{2}(g o \Delta u)(t) \\
\leq & \varepsilon E(t)+\varepsilon \frac{c^{\rho+2}}{(\rho+1)(\rho+2)}\left(\frac{6}{\ell} E(0)\right)^{1+\frac{\rho}{2}} E(t)+3 \varepsilon E(t) \\
& +\frac{3 \varepsilon}{\ell} E(t)+\frac{3 c}{(\rho+1)}(E(0))^{\rho} E(t)+\frac{3(1-\ell)}{(\rho+1)} c_{p} E(t) \\
& +3 E(t)+3(1-\ell) E(t) \\
\leq & c(1+\varepsilon) E(t)
\end{aligned}
$$

that is

$$
[N-c(1+\varepsilon)] E(t) \leq L(t) \leq[N+c(1+\varepsilon)] E(t) .
$$

By fixing $N$ large enough so that $N>c(1+\varepsilon)$, we obtain the desired result (5.23). For the proof of (5.24), since $g$ is positive and $g(0)>0$ then, for any $t_{0}>0$, we have

$$
\int_{0}^{t} g(s) d s \geq \int_{0}^{t_{0}} g(s) d s=g_{0}>0, \forall t \geq t_{0}
$$

By using (2.5), (5.8) and (5.11) then, for $t \geq t_{0}$, we have

$$
\begin{aligned}
L^{\prime}(t) \leq & \left(\frac{N}{2}-\frac{c}{\delta_{2}}\right)\left(g^{\prime} o \Delta u\right)(t)-\frac{g_{0}-\varepsilon}{\rho+1} \int_{\Omega}\left|u_{t}\right|^{\rho+2} d x \\
& -\left[\varepsilon \frac{\ell}{2}-\left(1+2(1-\ell)^{2}\right) \delta_{1}-\frac{\delta}{4}\right]\|\Delta u\|_{2}^{2} \\
& -\left[g_{0}-\varepsilon-\delta_{2}-c \delta_{2}(E(0))^{\rho}\right]\left\|\Delta u_{t}\right\|_{2}^{2} \\
& +c\left(\varepsilon+\delta_{1}+\frac{1}{\delta_{1}}+\frac{1}{\delta}\right)(g o \Delta u)(t) \\
& +c_{\epsilon_{0}, \delta}(g o \Delta u)^{\frac{1}{1+\epsilon_{0}}}(t)+\varepsilon k \int_{\Omega} u^{2} \ln |u| d x .
\end{aligned}
$$


Using the definition of $E(t)$, we obtain, for any $m>0$,

$$
\begin{aligned}
L^{\prime}(t) \leq & -m E(t)+\left(\frac{N}{2}-\frac{c}{\delta_{2}}\right)\left(g^{\prime} o \Delta u\right)(t)-\left(\frac{g_{0}-\varepsilon}{\rho+1}-\frac{m}{\rho+2}\right) \int_{\Omega}\left|u_{t}\right|^{\rho+2} d x \\
& -\left[\varepsilon \frac{\ell}{2}-\left(1+2(1-\ell)^{2}\right) \delta_{1}-\frac{\delta}{4}-\frac{m\left(1-g_{0}\right)}{2}\right]\|\Delta u\|_{2}^{2} \\
& -\left[g_{0}-\varepsilon-\delta_{2}-c \delta_{2}(E(0))^{\rho}-\frac{m}{2}\right]\left\|\Delta u_{t}\right\|_{2}^{2} \\
& +\left[c\left(\varepsilon+\delta_{1}+\frac{1}{\delta_{1}}+\frac{1}{\delta}\right)+\frac{m}{2}\right](g o \Delta u)(t) \\
& +c_{\epsilon_{0}, \delta}(g o \Delta u)^{\frac{1}{1+\epsilon_{0}}}(t)+\frac{m k}{4}\|u\|_{2}^{2} \\
& +\left(\varepsilon-\frac{m}{2}\right) k \int_{\Omega} u^{2} \ln |u| d x .
\end{aligned}
$$

Using the Logarithmic Sobolev inequality (2.7), we get, for $0<m<2 \varepsilon$,

$$
\begin{aligned}
L^{\prime}(t) \leq & -m E(t)+\left[\frac{N}{2}-\frac{c}{\delta_{2}}\right]\left(g^{\prime} o \Delta u\right)(t)-\left(\frac{g_{0}-\varepsilon}{\rho+1}-\frac{m}{\rho+2}\right) \int_{\Omega}\left|u_{t}\right|^{\rho+2} d x \\
& -\left[\varepsilon \frac{\ell}{2}-\left(1+2(1-\ell)^{2}\right) \delta_{1}-\frac{\delta}{4}-\frac{m\left(1-g_{0}\right)}{2}-\left(\varepsilon-\frac{m}{2}\right) \frac{k c_{p} a^{2}}{2 \pi}\right]\|\Delta u\|_{2}^{2} \\
& -\left(g_{0}-\varepsilon-\delta_{2}-c \delta_{2}(E(0))^{\rho}-\frac{m}{2}\right)\left\|\Delta u_{t}\right\|_{2}^{2} \\
& +\left[c\left(\varepsilon+\delta_{1}+\frac{1}{\delta_{1}}+\frac{1}{\delta}\right)+\frac{m}{2}\right](g o \Delta u)(t)+c_{\epsilon_{0}, \delta}(g o \Delta u)^{\frac{1}{1+\epsilon_{0}}}(t) \\
& -\left(\varepsilon-\frac{m}{2}\right) \frac{k}{2}\left(2(1+\ln a)-\ln \|u\|_{2}^{2}\right)\|u\|_{2}^{2}+\frac{m k}{4}\|u\|_{2}^{2} .
\end{aligned}
$$

At this point, we choose our constant carefully. First, we pick $0<\varepsilon<g_{0}$, then $\delta_{1}$, $\delta_{2}$ and $\delta$ small enough so that

$$
k_{1}:=\varepsilon \frac{\ell}{2}-\left(1+2(1-\ell)^{2}\right) \delta_{1}-\frac{\delta}{4}>0
$$

and

$$
k_{2}:=g_{0}-\varepsilon-\delta_{2}-c \delta_{2}(E(0))^{\rho}>0 .
$$

Then, $N$ sufficiently large so that

$$
N>c(1+\varepsilon) \quad \text { and } \quad \frac{N}{2}-\frac{c}{\delta_{2}} \geq 0
$$


Consequently, we get

$$
\begin{aligned}
L^{\prime}(t) \leq & -m E(t)-\left(\frac{g_{0}-\varepsilon}{\rho+1}-\frac{m}{\rho+2}\right) \int_{\Omega}\left|u_{t}\right|^{\rho+2} d x \\
& -\left[k_{1}-\frac{m\left(1-g_{0}\right)}{2}-\left(\varepsilon-\frac{m}{2}\right) \frac{k c_{p} a^{2}}{2 \pi}\right]\|\Delta u\|_{2}^{2} \\
& -\left(k_{2}-\frac{m}{2}\right)\left\|\Delta u_{t}\right\|_{2}^{2}+\left(c+\frac{m}{2}\right)(g o \Delta u)(t) \\
& +c_{\epsilon_{0}}(g o \Delta u)^{\frac{1}{1+\epsilon_{0}}}(t)+\frac{m k}{4}\|u\|_{2}^{2} \\
& -\left(\varepsilon-\frac{m}{2}\right) \frac{k}{2}\left(2(1+\ln a)-\ln \|u\|_{2}^{2}\right)\|u\|_{2}^{2} .
\end{aligned}
$$

Finally, we choose $m$ and $k$ small enough so that $m \leq \varepsilon$ (so $\left.\frac{m k}{4} \leq\left(\varepsilon-\frac{m}{2}\right) \frac{k}{2}\right)$,

$$
\begin{gathered}
\frac{g_{0}-\varepsilon}{\rho+1}-\frac{m}{\rho+2}>0, \\
k_{1}-\frac{m\left(1-g_{0}\right)}{2}-\left(\varepsilon-\frac{m}{2}\right) \frac{k c_{p} a^{2}}{2 \pi}>0
\end{gathered}
$$

and

$$
k_{2}-\frac{m}{2}>0
$$

we get

$$
\begin{aligned}
L^{\prime}(t) & \leq-m E(t)+c(g o \Delta u)(t)+c_{\varepsilon_{0}}(g o \Delta u)^{\frac{1}{1+\varepsilon_{0}}}(t) \\
& -\left(\varepsilon-\frac{m}{2}\right) \frac{k}{2}\left(1+2 \ln a-\ln \|u\|_{2}^{2}\right)\|u\|_{2}^{2} .
\end{aligned}
$$

Using (2.4), 2.5, 4.1), 4.6), 4.8) and (5.22, we have

$$
\ln \|u\|_{2}^{2} \leq \ln \left(\frac{4}{k} J(t)\right) \leq \ln \left(\frac{4}{k} E(t)\right) \leq \ln \left(\frac{4}{k} E(0)\right) \leq \ln \left(\frac{e \ell \pi}{k c_{p}}\right) .
$$

By taking $a$ satisfying

$$
\max \left\{e^{-\frac{3}{2}}, \sqrt{\frac{\ell \pi}{k c_{p}}}\right\}<a<\sqrt{\frac{2 \ell \pi}{k c_{p}}}
$$

(so (3.7) is satisfied), we guarantee

$$
1+2 \ln a-\ln \|u\|_{2}^{2} \geq 0 .
$$

Which completes the proof of 5.24.

Remarks 5.8. Using 2.1, 2.4, 4.1, 4.6) and 4.8, we have

$$
E(t)=J(t)+\frac{1}{\rho+2}\left\|u_{t}\right\|_{\rho+2}^{\rho+2} \geq J(t) \geq \frac{l}{6}\|\Delta u(t)\|_{2}^{2},
$$

then, using 2.5,

$$
\|\Delta u(t)\|_{2}^{2} \leq \frac{6}{l} E(t) \leq \frac{6}{l} E(0) .
$$


So, from 2.5 and using Young's inequality, we get

$$
\begin{aligned}
\left|E^{\prime}(t)\right| & =\frac{1}{2} g(t)\|\Delta u(t)\|_{2}^{2}-\frac{1}{2}\left(g^{\prime} o \Delta u\right)(t) \\
& \leq \frac{1}{2} g(t)\|\Delta u(t)\|_{2}^{2}-\int_{0}^{t} g^{\prime}(t-s)\left(\|\Delta u(t)\|_{2}^{2}+\|\Delta u(s)\|_{2}^{2}\right) d s \\
& \leq \frac{6}{l}\left(\frac{1}{2} g(t)+2 g(0)-2 g(t)\right) E(0) \\
& \leq c E(0) .
\end{aligned}
$$

Theorem 5.9. Let $\left(u_{0}, u_{1}\right) \in H_{0}^{2}(\Omega) \times H_{0}^{2}(\Omega), \epsilon \in(0,2 p-1)$ and $t_{0}>0$. Assume that $(A 1)-(A 3)$ and 4.5 hold. Then, for $k$ small enough, there exists a positive constant $K$ such that the solution of (1.1) satisfies

$$
E(t) \leq K\left(1+\int_{t_{0}}^{t} \xi^{2 p-1+\epsilon}(s) d s\right)^{\frac{-1}{2 p-2+\epsilon}}, \quad \forall t \geq t_{0} .
$$

Moreover, if there exist $\epsilon_{1} \in(0,2 p-1)$ and $t_{0}>0$ such that

$$
\int_{t_{0}}^{\infty}\left(1+\int_{t_{0}}^{t} \xi^{2 p-1+\epsilon_{1}}(s) d s\right)^{\frac{-1}{2 p-2+\epsilon_{1}}} d t<\infty,
$$

then, for any $r \in(0, p)$ and $t_{0}>0$, there exists a positive constant $K$ such that the solution of (1.1) satisfies

$$
E(t) \leq K\left(1+\int_{t_{0}}^{t} \xi^{p+r}(s) d s\right)^{\frac{-1}{p-1+r}}, \quad \forall t \geq t_{0} .
$$

Remarks 5.10. Using (5.34) and (5.35), we can easily show that

$$
\int_{0}^{+\infty} E(t) d t<+\infty
$$

Proof. We multiply 5.24 by $\xi(t)$ and use Corollary 5.4 and 5.33 to get, for any $t \geq t_{0}$,

$$
\begin{aligned}
\xi(t) L^{\prime}(t) & \leq-m \xi(t) E(t)+c\left(-E^{\prime}(t)\right)^{\frac{1}{2 p-1}}+c\left(-E^{\prime}(t)\right)^{\frac{1}{(2 p-1)\left(1+\epsilon_{0}\right)}} \\
\leq & -m \xi(t) E(t)+c\left(-E^{\prime}(t)\right)^{\frac{\epsilon_{0}}{(2 p-1)\left(1+\epsilon_{0}\right)}}\left(-E^{\prime}(t)\right)^{\frac{1}{(2 p-1)\left(1+\epsilon_{0}\right)}}+c\left(-E^{\prime}(t)\right)^{\frac{1}{(2 p-1)\left(1+\epsilon_{0}\right)}} \\
\leq & -m \xi(t) E(t)+c\left(-E^{\prime}(t)\right)^{\frac{1}{(2 p-1)\left(1+\epsilon_{0}\right)}}, \quad \forall t \geq t_{0} .
\end{aligned}
$$

Multiply the last inequality by $\xi^{\gamma}(t) E^{\gamma}(t)$, where $\gamma=(2 p-1)\left(1+\epsilon_{0}\right)-1$, and notice that $\xi^{\prime} \leq 0$ to obtain

$$
\xi^{\gamma+1}(t) E^{\gamma}(t) L^{\prime}(t) \leq-m \xi^{\gamma+1}(t) E^{\gamma+1}(t)+c(\xi E)^{\gamma}(t)\left(-E^{\prime}(t)\right)^{\frac{1}{\gamma+1}}, \quad \forall t \geq t_{0} .
$$

Use of Young's inequality, with $q=\gamma+1$ and $q^{*}=\frac{\gamma+1}{\gamma}$, gives, for any $\varepsilon^{\prime}>0$,

$$
\begin{aligned}
\xi^{\gamma+1}(t) E^{\gamma}(t) L^{\prime}(t) & \leq-m \xi^{\gamma+1}(t) E^{\gamma+1}(t)+c\left(\varepsilon^{\prime} \xi^{\gamma+1}(t) E^{\gamma+1}-c_{\varepsilon^{\prime}} E^{\prime}(t)\right) \\
& =-\left(m-\varepsilon^{\prime} c\right) \xi^{\gamma+1}(t) E^{\gamma+1}-c E^{\prime}(t), \quad \forall t \geq t_{0} .
\end{aligned}
$$

We then choose $0<\varepsilon^{\prime}<\frac{m}{c}$ and recall that $\xi^{\prime} \leq 0$ and $E^{\prime} \leq 0$, to get, for $c_{1}=m-\varepsilon^{\prime} c$,

$$
\left(\xi^{\gamma+1} E^{\gamma} L\right)^{\prime}(t) \leq \xi^{\gamma+1}(t) E^{\gamma}(t) L^{\prime}(t) \leq-c_{1} \xi^{\gamma+1}(t) E^{\gamma+1}(t)-c E^{\prime}(t), \quad \forall t \geq t_{0},
$$


which implies

$$
\left(\xi^{\gamma+1} E^{\gamma} L+c E\right)^{\prime}(t) \leq-c_{1} \xi^{\gamma+1}(t) E^{\gamma+1}(t), \quad \forall t \geq t_{0} .
$$

Let $F=\xi^{\gamma+1} E^{\gamma} L+c E$. Then $F \sim E$ (thanks to $(5.23)$ ) and

$$
F^{\prime}(t) \leq-c \xi^{\gamma+1}(t) F^{\gamma+1}(t)=-c \xi^{(2 p-1)\left(1+\epsilon_{0}\right)}(t) F^{(2 p-1)\left(1+\epsilon_{0}\right)}(t), \forall t \geq t_{0} .
$$

Integrating over $\left(t_{0}, t\right)$ and using the fact that $F \sim E$, we obtain (5.34) with $\epsilon=(2 p-1) \epsilon_{0}$.

To establish (5.36), we use the idea of Messaoudi and Al-Khulaifi [32. Let

$$
\eta(t)=\int_{0}^{t}\|\Delta u(t)-\Delta u(t-s)\|_{2}^{2} d s .
$$

Using (5.34), (5.32), (5.35) and (5.37), we have

$$
\begin{aligned}
\eta(t) \leq & 2 \int_{0}^{t}\left(\|\Delta u(t)\|_{2}^{2}+\|\Delta u(t-s)\|_{2}^{2}\right) d s \\
& \leq \frac{12}{l} \int_{0}^{t}(E(t)+E(t-s)) d s \\
& \leq \frac{24}{l} \int_{0}^{t} E(s) d s<\frac{24}{l} \int_{0}^{\infty} E(s) d s<\infty .
\end{aligned}
$$

This implies that

$$
\sup _{t>0} \eta^{1-\frac{1}{p}}(t)<\infty .
$$

Assume that $\eta(t)>0$. Then, because $\xi$ is nonincreasing, we find

$$
\xi(t)(g \circ \Delta u)(t) \leq \frac{\eta(t)}{\eta(t)} \int_{0}^{t}\left(\xi^{p}(s) g^{p}(s)\right)^{\frac{1}{p}}\|\Delta u(t)-\Delta u(t-s)\|_{2}^{2} d s .
$$

Applying Jensen's inequality to get

$$
\xi(t)(g \circ \Delta u)(t) \leq \eta(t)\left(\frac{1}{\eta(t)} \int_{0}^{t} \xi^{p}(s) g^{p}(s)\|\Delta u(t)-\Delta u(t-s)\|_{2}^{2} d s\right)^{\frac{1}{p}} .
$$

Therefore, using $(A 2)$ and $(5.39)$ we obtain

$$
\begin{aligned}
& \xi(t)(g \circ \Delta u)(t) \leq \eta^{1-\frac{1}{p}}(t)\left(\xi^{p-1}(0) \int_{0}^{t} \xi(s) g^{p}(s)\|\Delta u(t)-\Delta u(t-s)\|_{2}^{2} d s\right)^{\frac{1}{p}} \\
& \leq c\left(-g^{\prime} \circ \Delta u\right)^{\frac{1}{p}}(t),
\end{aligned}
$$

and then, according to 2.5 ,

$$
\xi(t)(g \circ \Delta u)(t) \leq c\left(-E^{\prime}(t)\right)^{\frac{1}{p}} .
$$

So, since $\xi$ is nonincreasing,

$$
\begin{aligned}
\xi(t)(g \circ \Delta u)^{\frac{1}{1+\epsilon_{0}}}(t)=\left(\xi^{\epsilon_{0}}(t) \xi(t)(g \circ \Delta u)(t)\right)^{\frac{1}{1+\epsilon_{0}}} \\
\quad \leq\left(\xi^{\epsilon_{0}}(0) \xi(t)(g \circ \Delta u)(t)\right)^{\frac{1}{1+\epsilon_{0}}} \\
\quad \leq c(\xi(t)(g \circ \Delta u)(t))^{\frac{1}{1+\epsilon_{0}}} \\
\quad \leq c\left(-E^{\prime}(t)\right)^{\frac{1}{p\left(1+\epsilon_{0}\right)}} .
\end{aligned}
$$


If $\eta(t)=0$, then $s \rightarrow \Delta u(s)$ is a constant function on $[0, t]$. Therefore

$$
(g \circ \Delta u)(t)=0,
$$

and hence (5.40) and (5.41) hold.

Now, multiplying (5.24) by $\xi(t)$ and using (5.33), 5.40) and (5.41) to find, for any $t \geq t_{0}($ as for $(5.38)$ ),

$$
\begin{aligned}
\xi(t) L^{\prime}(t) & \leq-m \xi(t) E(t)+c\left(-E^{\prime}(t)\right)^{\frac{1}{p}}+c\left(-E^{\prime}(t)\right)^{\frac{1}{p\left(1+\epsilon_{0}\right)}} \\
\leq & -m \xi(t) E(t)+c\left(-E^{\prime}(t)\right)^{\frac{\epsilon_{0}}{p\left(1+\epsilon_{0}\right)}}\left(-E^{\prime}(t)\right)^{\frac{1}{p\left(1+\epsilon_{0}\right)}}+c\left(-E^{\prime}(t)\right)^{\frac{1}{p\left(1+\epsilon_{0}\right)}} \\
\leq & -m \xi(t) E(t)+c\left(-E^{\prime}(t)\right)^{\frac{1}{p\left(1+\epsilon_{0}\right)}}, \quad \forall t \geq t_{0} .
\end{aligned}
$$

Inequality (5.38) with $2 p-1$ replaced by $p$ is exactely (5.42). Then, the proof of 5.36) can be completed as for the one of (5.34) (by taking $\gamma=p\left(1+\epsilon_{0}\right)-1$ and $\left.\epsilon=p \epsilon_{0}\right)$. This completes the proof of our main result.

Remarks 5.11. We note here that $2 p-2+\epsilon$ and $p-1+\epsilon$ can be arbitrary close to $2 p-2$ and $p-1$, respectively, since $\epsilon$ can be arbitrary close to zero. On the other hand, in the absence of the logarithmic "forcing" term $(k=0)$, the estimates 2.10) and (5.21) drop out and, consequently, 5.24) takes the form

$$
L^{\prime}(t) \leq-m E(t)+c(g \circ \Delta u)(t), \quad \forall t \geq t_{0} .
$$

In this case, we obtain the following result:

Theorem 5.12. Let $\left(u_{0}, u_{1}\right) \in H_{0}^{2}(\Omega) \times H_{0}^{2}(\Omega)$ and $t_{0}>0$. Assume that $(A 1)-$ (A2) hold. Then, there exists a positive constant $K$ such that the solution of (1.1) satisfies, for all $t \geq t_{0}$,

$$
E(t) \leq K e^{-\lambda \int_{t_{0}}^{t} \xi(s) d s} \quad \text { if } p=1
$$

and

$$
E(t) \leq K\left(1+\int_{t_{0}}^{t} \xi^{2 p-1}(s) d s\right)^{\frac{-1}{2 p-2}} \quad \text { if } 1<p<\frac{3}{2} .
$$

Moreover, if $1<p<\frac{3}{2}$ and

$$
\int_{0}^{\infty}\left(1+\int_{t_{0}}^{t} \xi^{2 p-1}(s) d s\right)^{\frac{-1}{2 p-2}} d t<\infty
$$

then

$$
E(t) \leq K\left(1+\int_{t_{0}}^{t} \xi^{p}(s) d s\right)^{\frac{-1}{p-1}}, \quad \forall t \geq t_{0} .
$$

\section{Acknowledgment}

The authors thank KFUPM for its continuous support. This work was funded by KFUPM under Project \#IN151023. This work was initiated during the visit of the second author to KFUPM in December 2016. 


\section{REFERENCES}

[1] Barrow J. and Parsons P., Inflationary models with logarithmic potentials, Phys. Rev., D 52, 5576-5587 (1995).

[2] Bartkowski K. and Gorka P., One-dimensional Klein-Gordon equation with logarithmic nonlinearities, J. Phys. A, 41(35), 355201, 11 pp. (2008).

[3] Benaissa A. and Guesmia A., Energy decay of solutions of a wave equation of $\phi$-Laplacian type with a general weakly nolinear dissipation, Elec. J. Diff. Equa., 109, 1-22 (2008).

[4] Bialynicki-Birula I. and Mycielski J., Wave equations with logarithmic nonlinearities, Bull. Acad. Polon. Sci. Ser. Sci. Math. Astronom. Phys., 23(4), 461-466 (1975).

[5] Bialynicki-Birula I. and Mycielski J., Nonlinear wave mechanics, Ann. Physics, 100(1-2), 62-93 (1976).

[6] Cavalcanti M., Domingos Cavalcanti V. and Ferreira J., Existence and uniform decay for nonlinear viscoelastic equation with strong damping, Math. Methods Appl. Sci., 24, 10431053 (2001).

[7] Cavalcanti M. and Oquendo H., Frictional versus viscoelastic damping in a semilinear wave equation, SIAM J. Control Optim., 42(4), 1310-1324 (2003).

[8] Cavalcanti M., Domingos Cavalcanti V., Lasiecka I. and Webler C., Intrinsic decay rates for the energy of a nonlinear viscoelastic equation modeling the vibrations of thin rods with variable density, Adv. Nonlinear Anal. (2016). http://dx.doi.org/10.1515/anona-2016-0027

[9] Cazenave T. and Haraux A., Equations d'evolution avec non-linearite logarithmique, Ann. Fac. Sci. Toulouse Math., 2(1), 21-51 (1980).

[10] Chen H., Luo P. and Liu G.W., Global solution and blow-up of a semilinear heat equation with logarithmic nonlinearity, J. Math. Anal. Appl., 422, 84-98 (2015).

[11] Chen W. and Zhou Y., Global nonexistence for a semilinear Petrovsky equation, Nonlinear Analysis A, vol. 70, no. 9, 3203-3208 (2009).

[12] Enqvist K. and McDonald J., Q-balls and baryogenesis in the MSSM, Phys. Lett. B 425, 309-321 (1998).

[13] Gorka P., Logarithmic Klein-Gordon equation, Acta Phys. Polon. B, 40(1), 59-66 (2009).

[14] Gorka P., Prado H. and Reyes E. G., Nonlinear equations with infinitely many derivatives, Complex Anal. Oper. Theory, 5, no. 1, 313-323 (2011).

[15] Gross L., Logarithmic Sobolev inequalities, Amer. J. Math., 97(4), 1061-1083 (1975).

[16] Guesmia A., Existence globale et stabilisation interne non linéaire d'un système de Petrovsky, Bull. Belg. Math. Soc., 5, 583-594 (1998).

[17] Han X., Global existence of weak solutions for a logarithmic wave equation arising from Q-ball dynamics. Bull. Korean Math. Soc., 50(1), 275-283 (2013).

[18] Han X. and Wang M., General decay of energy for a viscoelastic equation with nonlinear damping, Math. Methods Appl. Sci., 32 (3), 346-358 (2009).

[19] Han X. and Wang M., General Decay Estimate of Energy for the Second Order Evolution Equations with Memory, Act Appl. Math., 110, 194-207 (2010).

[20] Hiramatsu T., Kawasaki M. and Takahashi F., Numerical study of Q-ball formation in gravity mediation, Journal of Cosmology and Astroparticle Physics, no. 6, 008 (2010).

[21] Komornik V., On the nonlinear boundary stabilization of Kirchoff Plates, NoDEA, 1, 323-337 (1994).

[22] Lagnese J., Boundary Stabilization of Thin Plates, SIAM, Philadelphia, 1989.

[23] Lagnese J., Asymptotic energy estimates for Kirchhoff plates subject to weak viscoelastic damping, International Series of Numerical Mathematics, vol. 91. Birhauser: Verlag, Bassel, (1989).

[24] Lasiecka I. and Tataru D., Uniform boundary stabilization of semilinear wave equation with nonlinear boundary damping, Differential Integral Equations, 6 (6), 507-533 (1993).

[25] Lasiecka I., Exponential decay rates for the solutions of Euler-Bernoulli moments only, J. Differential Equations 95, 169-182 (1992).

[26] Lions J., Quelques methods de resolution des probléms aux limites non lineaires, Dunod Gauthier-Villars, Paris, (1969).

[27] Liu W., General decay and blow up of solution for a quasilinear viscoelastic equation with a nonlinear source, Nonlinear Anal., 73, 1890-1904 (2010). 
[28] Liu W., General decay rate estimate for a viscoelastic equation with weakly nonlinear timedependent dissipation and source terms, J. Math. Phys., 50 (11), Art. No. 113506, pp. 17 (2009).

[29] Messaoudi S., Global existence and nonexistence in a system of Petrovsky, Journal of Mathematical Analysis and Applications, vol. 265, no. 2, 296-308 (2002).

[30] Messaoudi S., General decay of solution energy in a viscoelastic equation with a nonlinear source, Nonlinear Anal., 69, 2589-2598 (2008).

[31] Messaoudi S., General decay of solutions of a viscoelastic equation, J. Math. Anal. App., 341, 1457-1467 (2008).

[32] Messaoudi S. and Al-Khulaifi W., General and optimal decay for a quasilinear viscoelastic equation, Applied Mathematics Letters, 66, 16-22 (2017).

[33] Messaoudi S. and Tatar N.-E, Global existence asymptotic behavior for a non-linear viscoelastic problem, Math. Methods Sci. Res., 7(4), 136-149 (2003).

[34] Messaoudi S. and Tatar N.-E, Global existence and uniform stability of solutions for a quasilinear viscoelastic problem, Math. Methods Appl. Sci., 30, 665-680 (2007).

[35] Messaoudi S. and Tatar N.-E, Exponential and polynomial decay for a quasilinear viscoelastic equation, Nonlinear Anal., TMA 68, 785-793 (2007).

[36] Messaoudi S. and Mustafa M., A general stability result for a quasilinear wave equation, Nonlin. Anal., (14), 1854-1864 (2013).

[37] Mu $\tilde{n}_{\mathrm{oz}}$ Rivera J., Lapa E. C. and Barreto R., Decay rates for viscoelastic paltes with memory, Journal of Elasticity, 44, 61-87 (1996).

[38] Santos M., Junior F., A boundary condition with memory for Kirchoff plates equations, Appl. Math. Comput., 148, 475-496 (2004).

[39] Vladimirov V. S., The equation of the p-adic open string for the scalar tachyon field, Izv. Math., 69, no. 3, 487-512 (2005). 